\title{
Iterative Multiuser Detection, Macrodiversity Combining, and Decoding for the TDMA Cellular Uplink
}

\author{
Matthew C. Valenti, Member, IEEE, and Brian D. Woerner, Member, IEEE
}

\begin{abstract}
A soft-input soft-output (SISO) multiuser detector (MUD) suitable for inclusion in iterative processing architectures is presented and applied to the detection of the coded time division multiple access (TDMA) cellular uplink. A SISO-MUD processor is located at each base station in the network, and adjacent base stations share information concerning the mobiles they serve. Because the MUD outputs are soft, they are suitable for postdetection macrodiversity combining. The combined signals are then passed to a SISO forward error correction (FEC) decoder, and the soft outputs are fed back to the multiuser detectors. Processing continues in an iterative fashion in accordance with the turbo principle. Simulation results are presented that indicate that use of such a scheme enables cellular systems to be overloaded with more than just one cochannel user per cell at the price of a minimal loss in signal-to-noise ratio (SNR). The proposed implementation assumes the availability of both perfect channel state information and a high capacity backhaul.
\end{abstract}

Index Terms-Channel coding, diversity reception, multiuser detection, turbo principle.

\section{INTRODUCTION}

$\mathbf{F}$ ADING and multiple-access interference (MAI) are two factors that limit the performance of multiple-access networks. There exists a host of techniques to mitigate these problems either individually or jointly. In particular, multiuser detection (MUD) can be used to combat MAI, while receive antenna diversity can be used to combat fading. The combination of strong forward error correction (FEC) codes and interleaving can be used to combat both MAI and fading. However, until recently, the various signal processing operations (MUD, FEC decoding, diversity combining, etc.) tended to operate in isolation from one another. The lack of cooperation among processes in conventional receiver design translates into performance that is far from ideal. Recently, several authors have proposed techniques for performing iterative detection and decoding of coded multiple-access signals [1], [2]. These algorithms are an ex-

Manuscript received December 15, 2000; revised May 24, 2001. This work was supported by the Office of Naval Research under Grant N00014-00-1-0655, the MPRG Industrial Affiliates Foundation, and the Defense Advanced Research Agency (DARPA) through the GLoMo program. This work was presented in part at the Second International Symposium on Turbo Codes and Related Applications, Brest, France, September 2000.

M. C. Valenti is with the Lane Department of Computer Science and Electrical Engineering, West Virginia University, Morgantown, WV 26506-6109 USA (e-mail: mvalenti@csee.wvu.edu).

B. D. Woerner is with the Mobile and Portable Radio Research Group, Virginia Polytechnic Institute and State University, Blacksburg, VA 24061-0350 USA (e-mail: woerner@vt.edu).

Publisher Item Identifier S 0733-8716(01)07224-9. tension of the turbo-processing concept originally proposed in conjunction with turbo codes [3]. Likewise, the combination of diversity reception and multiuser detection has attracted attention [4]. In this paper, we apply the turbo concept to the iterative combination of all three processes: diversity reception, multiuser detection, and error correction coding with interleaving. We will show by example and simulation that such a strategy is particularly well suited for coded time division multiple-access (TDMA) networks.

While MUD is usually considered for use with direct sequence code division multiple-access (DS-CDMA), it can also be used to improve the performance of TDMA cellular systems [5], [6]. When used for DS-CDMA systems, the objective of MUD is to jointly detect signals that originate from the same cell (i.e., intracell interference). When used for TDMA systems, however, the objective of MUD is to jointly detect the desired signal and cochannel interferers originating from nearby cells (i.e., intercell interference). The key difference is that with DS-CDMA all the signals of interest (SOI) are in the same cell and, hence, all serviced by the same base station (BS), while with TDMA, the SOI are more distributed and served by different BSs. With DS-CDMA, there is little to be gained by having neighboring BSs share information (aside from soft handoff). However, with TDMA, there is the potential for an enormous performance gain by allowing adjacent BSs to share information.

The approach we take regarding TDMA multiuser detection is as follows. First, each BS in a group of cochannel cells performs soft-output multiuser detection of the desired signal (originating from its cell) and the interfering signals (originating from the other cells in the group). The multiuser detector thus produces an estimate [in the form of a log-likelihood ratio (LLR)] of each mobile in the group. The LLRs for each user are then summed across the BSs, which in effect produces a diversity combined signal. Because the BSs, and hence their antennas, are widely separated, this is a form of macrodiversity (as opposed to microdiversity, where the antennas are all in close proximity). The approach of combining the outputs of multiple BSs considered here is similar to the multiply-detected macrodiversity (MDM) scheme of [7], which is a method of combining the hard bit decisions made by conventional receivers at multiple BSs. Our method differs from [7] in that a soft-output multiuser detector is employed at each BS and diversity combining uses LLRs rather than hard bit decisions.

Once multiuser detection and postdetection combining has taken place, the signal may be deinterleaved and decoded. If the 
decoder also produces soft outputs, then this output may be reinterleaved and fed back to the multiuser detectors to be used as a priori information. Thus, the whole process of multiuser detection, macrodiversity combining, and decoding can be iterated a second or third time (or more).

It should be noted that the proposed system places an extra burden on the backhaul links. Since soft information is now shared among BSs, a wider data path will be required from the individual BSs to the base station controller (BSC). If the multiuser detectors are physically located at the BSs, then iterative processing will require soft information to be passed back from the BSC to the BSs. However, a better solution would be for the BSs to simply pass the matched filter outputs to the BSC. This would allow both multiuser detection and channel decoding to be performed at the BSC, which in turn would eliminate the need for a return path from the BSC to each BS.

We continue this paper with a mathematical description of the system. Next, an optimum soft-input soft-output (SISO) multiuser detector is derived which is suitable for use in TDMA systems. The soft-output MUD algorithm bears some resemblance to the one presented in [8], although our exposition is more detailed and uses the concept of noise whitening. The basis of the algorithm is the optimal multiuser detection algorithm of [9] which has been modified to produce soft-outputs by utilizing the BCJR algorithm of [10] rather than the Viterbi algorithm. We presented an overview of this algorithm in [11]. Although the complexity of this multiuser detector is exponential in the number of users, we are justified in using it because the number of cochannel users in a TDMA network is typically rather low. Next, we discuss how the outputs of the multiuser detectors of adjacent BSs can be combined. At this point, we show some simulation results to illustrate the potential of using multiuser detection with post-detection macrodiversity combining in a TDMA uplink. The results that we show here are extensions of our preliminary work presented in [12] and [13]. Next, we discuss how coding can be used to improve performance further and suggest an iterative structure for combined multiuser detection and FEC decoding. Simulation results are presented to illustrate the performance of iterative MUD and FEC, and are extensions of our work presented in [5] and [14]. Finally, we conclude with a discussion of the limitations of our approach and suggestions for future work.

Throughout this paper, scalars are denoted by italicized lowercase letters (e.g., $c$ ), vectors by boldface lowercase letters (e.g., c), and matrices by boldface uppercase letters (e.g., C). The $i$ th element of $\mathbf{c}$ is denoted $\mathbf{c}_{i}$ and the $(i, j)$ th element of $\mathbf{C}$ is denoted $\mathbf{C}_{i, j}$. To be consistent with the literature, $\boldsymbol{\Lambda}$ is used to denote a vector of LLRs. The complex conjugate of $c$ is denoted $c^{*}$ and $\mathbf{C}^{-\mathrm{T}}$ is the transpose of the inverse of $\mathbf{C}$.

\section{SYSTEM MODEL}

In this section, we briefly outline a discrete-time model of a multiple-access communication system comprised of $K$ trans- mitters and $M$ receivers. The system model is similar to the one used in [15] except that we have allowed for multiple receivers and constrain all the TDMA users to transmit with the same waveform.

\section{A. Transmitter}

The input to transmitter $k, 1 \leq k \leq K$ is a set $\left\{m_{k, i}\right\}, 0 \leq$ $i \leq L-1$ of random binary data $m_{k, i} \in\{0,1\}$. The bits are encoded by a rate $r$ encoder. The code bits $\left\{x_{k, l}\right\}, 0 \leq l \leq$ $L / r-1$ are reordered by an interleaving function $\alpha_{k}$ to form the set of interleaved code bits $\left\{\bar{x}_{k, l}\right\}$ where $\bar{x}_{k, \alpha_{k}(l)}=x_{k, l}$.

The interleaved code bits are passed to a signal mapper, which creates a stream of symbols $\left\{v_{k, n}\right\}, 0 \leq n \leq N-1$. For purposes of establishing a discrete-time model, it is convenient to place the symbols into a vector in a "round-robin" fashion according to

$$
\mathbf{v}=\left[\begin{array}{lllllll}
v_{1,0} & v_{2,0} & \ldots & v_{K, 0} & v_{1,1} & \ldots & v_{K, N-1}
\end{array}\right]^{\mathrm{T}} .
$$

The symbols are passed through a pulse-shaping filter with impulse response $g(t)$ producing the sequence

$$
s_{k}(t)=\sum_{n=0}^{N-1} v_{k, n} g\left(t-n T_{s}\right) .
$$

The average energy per symbol of the modulated sequence is $E_{s}$. Note that, because we are restricting our attention to TDMA systems, all users in the system are characterized with the same impulse response. Had we considered DS-CDMA systems, then $g(t)$ would not only be a function of the chip-shaping waveform but would also be a function of the different users' signature sequences.

\section{B. Channel Model}

The signal transmitted from each user is passed through a Rayleigh flat-fading channel. The signals from the $K$ transmitters arrive asynchronously at receiver $m$, giving rise to the received signal

$$
r_{m}(t)=\sum_{k=1}^{K} c_{m, k}(t) s_{k}\left(t-\tau_{m, k}\right)+n_{m}(t)
$$

where

$$
\begin{aligned}
& \begin{array}{l}
n_{m}(t) \quad \begin{array}{l}
\text { complex white Gaussian noise process with two- } \\
\text { sided noise spectral density } N_{o} / 2 ;
\end{array} \\
c_{m, k}(t) \quad \begin{array}{l}
\text { complex fading process; } \\
\tau_{m, k}
\end{array} \\
\text { relative propagation delay, which is assumed to be } \\
\text { less than one symbol period } 0 \leq \tau_{m, k}<T_{s} \text { and } \\
\text { sorted in ascending order } \tau_{m, i} \leq \tau_{m, j} \text { for } i<j .
\end{array} \\
& \text { The channel coefficients corresponding to receiver } m \text { are } \\
& \text { placed into a vector in round robin fashion, see }(4) \text { at the } \\
& \text { bottom of the page, where } c_{m, k, n}=c_{m, k}\left(n T_{s}\right) \text { are the } \\
& \text { sampled values of the channel gain between transmitter } k \text { and }
\end{aligned}
$$

$$
\mathbf{c}^{(m)}=\left[\begin{array}{lllllll}
c_{m, 1,0} & c_{m, 2,0} & \ldots & c_{m, K, 0} & c_{m, 1,1} & \ldots & c_{m, K, N-1}
\end{array}\right]^{\mathrm{T}}
$$


receiver $m$. The gains are also placed into a diagonal matrix $\mathbf{C}^{(m)}=\operatorname{diag}\left\{\mathbf{c}^{(m)}\right\}$.

The discrete-time model requires a set of crosscorrelations at the channel output, which are defined for $i<j$

$$
\begin{aligned}
& \rho_{i, j}^{(m)}=\frac{1}{E_{s}} \int_{\delta_{j, i}^{(m)}}^{T_{s}} g(t) g\left(t-\delta_{j, i}^{(m)}\right) d t \\
& \rho_{j, i}^{(m)}=\frac{1}{E_{s}} \int_{0}^{\delta_{j, i}^{(m)}} g(t) g\left(t-T_{s}-\delta_{j, i}^{(m)}\right) d t
\end{aligned}
$$

where $\delta_{j, i}^{(m)}=\tau_{m, j}-\tau_{m, i}$. The crosscorrelations are stored in a symmetric matrix

$$
\mathbf{R}^{(m)}[0]=\left[\begin{array}{cccc}
1 & \rho_{1,2}^{(m)} & \cdots & \rho_{1, K}^{(m)} \\
\rho_{1,2}^{(m)} & 1 & \cdots & \rho_{2, K}^{(m)} \\
\vdots & \vdots & & \vdots \\
\rho_{1, K}^{(m)} & \rho_{2, K}^{(m)} & \cdots & 1
\end{array}\right]
$$

and an upper triangular matrix

$$
\mathbf{R}^{(m)}[1]=\left[\begin{array}{ccccc}
0 & \rho_{2,1}^{(m)} & \rho_{3,1}^{(m)} & \ldots & \rho_{K, 1}^{(m)} \\
0 & 0 & \rho_{3,2}^{(m)} & \ldots & \rho_{K, 2}^{(m)} \\
0 & 0 & 0 & \ldots & \rho_{K, 3}^{(m)} \\
\vdots & \vdots & \vdots & & \vdots \\
0 & 0 & 0 & \ldots & 0
\end{array}\right] .
$$

Finally, a $(K N \times K N)$ global correlation matrix [see (9) at the bottom of the page] is defined with nonzero entries only on diagonals $-K$ through $K$.

\section{Receiver}

The front end of receiver $m$ contains a bank of $K$ matched filters whose output for a particular user $k^{\prime}$ is

$$
y_{m, k^{\prime}, n}=\frac{1}{E_{s}} \int_{0}^{\mathrm{T}_{\mathrm{s}}} g(t) y_{m}\left(t+\tau_{m, k^{\prime}}+n T_{s}\right) d t .
$$

If the matched filter coefficients are placed into a vector $y^{(m)}$, which is defined the same way as $\mathbf{c}^{(m)}$ only with $c_{m, k, n}$ replaced with $y_{m, k, n}$, then the output of the matched filter can be expressed as

$$
\mathbf{y}^{(m)}=\mathbf{R}^{(m)} \mathbf{C}^{(m)} \mathbf{v}+\mathbf{n}^{(m)} .
$$

In the above expression, $\mathbf{n}^{(m)}$ is a Gaussian noise vector with autocorrelation $\sigma^{2} \mathbf{R}^{(m)}$, with $\sigma^{2}=N_{o} /\left(2 E_{s}\right)$.

\section{Whitened Matched Filter}

Because the noise samples are colored, it is cumbersome to compute the exact LLRs of the symbols using the statistic $\mathbf{y}^{(m)}$. However, because $\mathbf{R}^{(m)}$ is positive definite, there exists a lower triangular matrix $\mathbf{F}^{(m)}$ with positive diagonal elements such that $\mathbf{R}^{(m)}=\left(\mathbf{F}^{(m)}\right)^{\mathrm{T}} \mathbf{F}^{(m)}$ [15]. The matrix $\mathbf{F}^{(m)}$ is found by performing a Cholesky decomposition of $\mathbf{R}^{(m)}$, and only has nonzero entries in diagonals $-\mathbf{K}$ through 0 . The whitened matched filter outputs are then

$$
\begin{aligned}
\overline{\mathbf{y}}^{(m)} & =\left(\mathbf{F}^{(m)}\right)^{-\mathrm{T}} \mathbf{y}^{(m)} \\
& =\mathbf{F}^{(m)} \mathbf{C}^{(m)} \mathbf{v}+\overline{\mathbf{n}}^{(m)}
\end{aligned}
$$

where $\overline{\mathbf{n}}^{(m)}$ is a white Gaussian noise process with variance $\sigma^{2}=E_{s} /\left(2 N_{o}\right)$.

By taking advantage of the structure of $\mathbf{F}^{(m)}$ and $\mathbf{C}^{(m)}$, which are both sparse matrices, (12) can be expressed more efficiently as

$$
\overline{\mathbf{y}}_{i}^{(m)}=\sum_{j=0}^{\min (i, K-1)} \mathbf{F}_{i, i-j}^{(m)} \mathbf{c}_{i-j}^{(m)} \mathbf{v}_{i-j}+\overline{\mathbf{n}}_{i}^{(m)} .
$$

A benefit of whitening the matched filter outputs is that the decision statistic $\overline{\mathbf{y}}_{i}^{(m)}$ is only a function of the current and past $K-1$ symbols observed through noise. This is in contrast to the unwhitened statistic $\mathbf{y}_{i}^{(m)}$, which is a noisy function of not only the current and past $K-1$ symbols, but also the next $K-1$ symbols. This property of the whitened matched filter output will be exploited in the development of the SISO MUD algorithm in the next section.

\section{SISO MUD ALGORITHM}

The SISO MUD algorithm for receiver $m$ computes the LLR

$$
\boldsymbol{\Lambda}_{i}^{(m)}=\ln \frac{P\left[\mathbf{v}_{i}=+1 \mid \overline{\mathbf{y}}^{(m)}\right]}{P\left[\mathbf{v}_{i}=-1 \mid \overline{\mathbf{y}}^{(m)}\right]} .
$$

By considering the whitened MAI channel to be a timevarying Markov process, a suitable SISO MUD algorithm can be developed by generalizing the BCJR algorithm of [10]. To see this, first define a one-to-one mapping between the state of the Markov process and the set of $K-1$ past symbols

$$
s_{i} \Longleftrightarrow\left\{\mathbf{v}_{i-1}, \mathbf{v}_{i-2}, \ldots, \mathbf{v}_{i-K}\right\} .
$$

Likewise, there is a one-to-one mapping between the state transition $s_{i} \rightarrow s_{i+1}$ and the union of the sets containing the past $K-1$ symbols and the set containing the current symbol

$$
\left(s_{i} \rightarrow s_{i+1}\right) \Longleftrightarrow\left\{\mathbf{v}_{i}, \mathbf{v}_{i-1}, \mathbf{v}_{i-2}, \ldots, \mathbf{v}_{i-K}\right\} .
$$


Finally, define a function that reconstructs the noiseless whitened matched filter output, given the state transition

$$
f_{i}^{(m)}\left(s_{i} \rightarrow s_{i+1}\right)=\sum_{j=0}^{\min (i, K-1)} \mathbf{F}_{i, i-j}^{(m)} \mathbf{c}_{i-j}^{(m)} \mathbf{v}_{i-j} .
$$

Now define a branch metric

$\lambda_{i}\left(s_{i} \rightarrow s_{i+1}\right)=\ln P\left[\overline{\mathbf{y}}_{i}^{(m)} \mid i, s_{i} \rightarrow s_{i+1}\right]+\ln P\left[s_{i+1} \mid s_{i}\right]$.

Note that this branch metric can depend on the index $i$. The term $P\left[\overline{\mathbf{y}}_{i}^{(m)} \mid i, s_{i} \rightarrow s_{i+1}\right]$ is Gaussian with mean $f_{i}^{(m)}\left(s_{i} \rightarrow s_{i+1}\right)$ and variance $\sigma^{2}=N_{o} / 2 E_{s}$, and thus

$$
\begin{aligned}
\ln P & {\left[\mathbf{y}_{i}^{(m)} \mid i, s_{i} \rightarrow s_{i+1}\right] } \\
= & -\frac{1}{2} \ln \left(\frac{\pi N_{o}}{E_{s}}\right) \\
& \quad-\frac{E_{s}}{N_{o}}\left|\overline{\mathbf{y}}_{i}^{(m)}-f_{i}^{(m)}\left(s_{i} \rightarrow s_{i+1}\right)\right|^{2} .
\end{aligned}
$$

The transition probability $P\left[s_{i+1} \mid s_{i}\right]$ is identical to the probability $P\left[\mathbf{v}_{i}\right]$ of the symbol that causes the transition. In situations that the multiuser detector is working in isolation, without any side information, it is generally assumed that the symbols are equiprobable. However, if there is side information available from another process, such as a channel decoder, then the SISO MUD algorithm can incorporate this information as an a priori input $\mathbf{z}_{i}$. For BPSK, the a priori input $\mathbf{z}_{i}$ can be expressed as a $\log$-likelihood ratio, and is related to the transition probability by

$$
\begin{aligned}
P\left[s_{i+1} \mid s_{i}\right] & =P\left[\mathbf{v}_{i}:\left(s_{i} \rightarrow s_{i+1}\right)\right] \\
& = \begin{cases}\frac{e^{\mathbf{z}_{i}}}{1+e^{\mathbf{z}_{i}}} & \text { for } \mathbf{v}_{i}=1 \\
\frac{1}{1+e^{\mathbf{z}_{i}}} & \text { for } \mathbf{v}_{i}=-1\end{cases}
\end{aligned}
$$

and thus

$$
\ln P\left[s_{i+1} \mid s_{i}\right]=\frac{\mathbf{z}_{i} \mathbf{v}_{i}}{2}+\frac{\mathbf{z}_{i}}{2}-\ln \left(1+e^{\mathbf{z}_{i}}\right) .
$$

Substituting (19) and (21) into (18) yields

$$
\begin{aligned}
\lambda_{i}\left(s_{i} \rightarrow s_{i+1}\right)= & \frac{\mathbf{z}_{i} \mathbf{v}_{i}}{2} \\
& -\frac{E_{s}}{N_{o}}\left|\overline{\mathbf{y}}_{i}^{(m)}-f_{i}^{(m)}\left(s_{i} \rightarrow s_{i+1}\right)\right|^{2}+\eta
\end{aligned}
$$

with

$$
\eta=\frac{\mathbf{z}_{i}}{2}-\ln \left(1+e^{\mathbf{z}_{i}}\right)-\frac{1}{2} \ln \left(\frac{\pi N_{o}}{E_{s}}\right) .
$$

The log-MAP algorithm [16] can now be used to calculate the LLR (14). When used to perform SISO multiuser detection, the branch metric (22) is used, with $\eta$ set to any arbitrary value (either zero or a normalization constant chosen to improve numerical stability).

\section{DistRibuted MultiUSER DeTECTION}

The SISO MUD algorithm can be applied to the problem of jointly detecting multiple-access signals observed by multiple receivers. This is a form of diversity combining, with the LLR outputs of the multiuser detectors at the $M$ receivers combined in an appropriate manner.

\section{A. Diversity Combining}

Maximal ratio combining (MRC) the output of multiple receivers is a classic means of improving performance in a fading channel. Given the set of matched filter outputs and channel gains, the maximal ratio combined statistic without multiuser detection is

$$
\mathbf{y}_{i}=\sum_{m=1}^{M} \mathbf{y}_{i}^{(m)}\left(\mathbf{c}_{i}^{(m)}\right)^{*} .
$$

The MRC rule (24) provides optimal symbol decisions when there is no MAI. In a MAI channel, it is desirable to first separate the users before applying the diversity combining rule. With distributed multiuser detection, the goal is to produce the LLR

$$
\boldsymbol{\Lambda}_{i}=\ln \frac{P\left[\mathbf{v}_{i}=+1 \mid \overline{\mathbf{y}}^{(1)}, \ldots, \overline{\mathbf{y}}^{(M)}\right]}{P\left[\mathbf{v}_{i}=-1 \mid \overline{\mathbf{y}}^{(1)}, \ldots, \overline{\mathbf{y}}^{(M)}\right]}
$$

that is, to find the LLR given the observations at all $M$ receivers. For independent observations, (25) becomes

$$
\begin{aligned}
\boldsymbol{\Lambda}_{i} & =\ln \frac{\prod_{m=1}^{M} P\left[\mathbf{v}_{i}=+1 \mid \overline{\mathbf{y}}^{(m)}\right]}{\prod_{m=1}^{M} P\left[\mathbf{v}_{i}=-1 \mid \overline{\mathbf{y}}^{(m)}\right]} \\
& =\sum_{m=1}^{M} \ln \frac{P\left[\mathbf{v}_{i}=+1 \mid \overline{\mathbf{y}}^{(m)}\right]}{P\left[\mathbf{v}_{i}=-1 \mid \overline{\mathbf{y}}^{(m)}\right]} \\
& =\sum_{m=1}^{M} \boldsymbol{\Lambda}_{i}^{(m)} .
\end{aligned}
$$

Thus, the combining rule for distributed MUD is to simply add the LLR outputs of the multiuser detectors at the $M$ receivers.

\section{B. Distributed MUD for the TDMA Uplink}

In TDMA systems, each mobile station (MS) transmits during a preassigned time slot using a designated carrier frequency. When the BS uses an isotropic antenna, only one MS in that cell is allowed to transmit in a particular time/frequency slot. Adjacent cells generally do not use the same time/frequency slots. However, distant cells reuse the time/frequency slots. For each time/frequency slot, each BS receives the transmission from one MS within the cell, along with interfering transmissions from other clusters. The performance of the cellular system is limited by the out-of-cell interference and the effects of the fading channel. With isotropic antennas, the interference is dominated by the six adjacent clusters that make up the first tier of cochannel transmitters.

The capacity of a cellular system can be increased by reducing the number of cells in a cluster. A common method for achieving this is to use 120-degree sectorized antennas, which reduces the number of first-tier interferers to two. Because of the reduced interference, it is generally possible to decrease the number of cells per cluster to three or four. With 120-degree sectorized antennas, it is appropriate to model the system as a grid of hexagons with BSs located on three equally-spaced corners of the hexagon. An example layout with three cells per cluster 


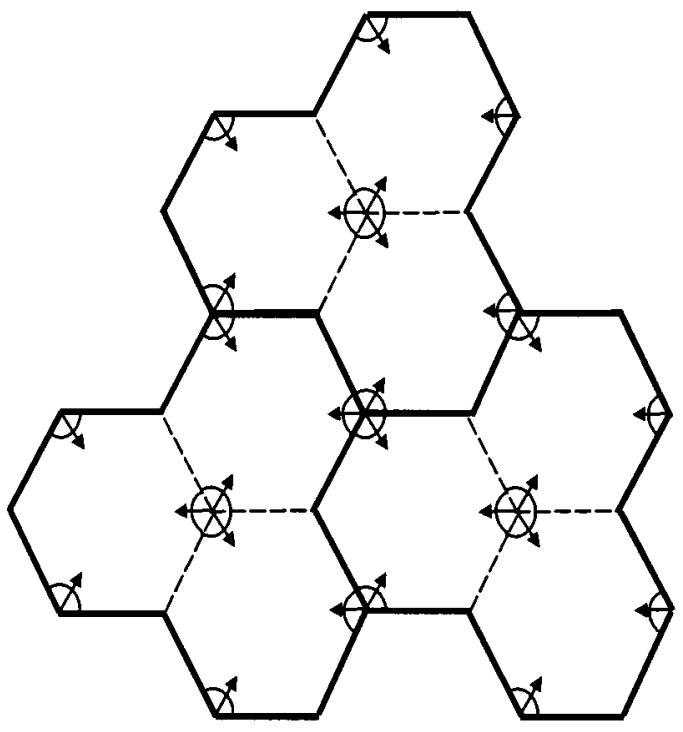

Fig. 1. Topology of an edge-excited cellular system with 120-degree sectorized antennas and three hexagonal cells per cluster.

and 120-degree sectorized antennas is shown in Fig. 1. In this figure, the center of each antenna beam pattern is indicated by an arrow. For each time/frequency slot assigned to the cell, there will be one mobile in the cell, which will be served by the closest of the three BSs. However, because of the proximity of the two other BS associated with the cell, a macrodiversity effect can be achieved on the uplink by MRC the outputs of all three BSs. This is particularly beneficial when the mobile is near the center of the cell, and thus, the received signal powers at the three BS are approximately equal.

With the judicious use of both multiuser detection and macrodiversity, it is possible to overload the cell with more than one cochannel user at the cost of a slight reduction in SNR, the need for a wider data path from BS to BSC, and the need for accurate channel estimates. Thus, the capacity of a TDMA cellular system with 120-degree sectorized antennas can be greatly increased by incorporating a SISO MUD algorithm at each BS and combining the MUD outputs of all three BSs located on the corner of each cell. We shall now illustrate the potential gains of such a scheme through simulation.

\section{Simulation Study: Uncoded Case}

Consider a TDMA system with topology shown in Fig. 1. Within each cell, there are $K$ cochannel users that share the same time/frequency slot. The $K$ transmitters are uncoded, and transmit frames of $L=100$ BPSK symbols. The channel is a fully-interleaved Rayleigh flat-fading channel ${ }^{1}$. The signals arrive at each BS asynchronously with relative delay

$$
\tau_{m, k}=\left(\frac{k-1}{K}\right) T_{s} .
$$

Note that in practice the relative delays may be any arbitrary value. However, our goal here is merely to illustrate the poten-

\footnotetext{
${ }^{1}$ Fully interleaved implies that the autocorrelation of the channel gain $c(t)$ is $R_{c}(\tau)=0.5 \delta(\tau)$ in each complex dimension.
}

tial benefits of the combination of multiuser detection with postdetection macrodiversity combining.

Four reception techniques are compared.

a) The conventional matched filter with hard bit decisions produced by hard limiting the phase corrected matched filter output

$$
\hat{\mathbf{v}}_{i}=\operatorname{sign}\left\{\mathbf{y}_{i}^{\left(m^{\prime}\right)} \exp \left(-j \angle \mathbf{c}_{i}^{\left(m^{\prime}\right)}\right)\right\}
$$

where $m^{\prime}$ is the BS closest to the MS that transmitted the symbol $\mathbf{v}_{i}$ and the $\exp \left(-j \angle \mathbf{c}_{i}^{\left(m^{\prime}\right)}\right)$ term is necessary to coherently detect the BPSK signal.

b) MRC the matched filter outputs of the three BSs that border the cell according to (24), followed by hard limiting the MRC statistic

$$
\hat{\mathbf{v}}_{i}=\operatorname{sign}\left\{\sum_{m=1}^{M} \mathbf{y}_{i}^{(m)}\left(\mathbf{c}_{i}^{(m)}\right)^{*}\right\}
$$

where the $M=3$ receivers are the three BSs that border the cell.

c) Performing multiuser detection at each BS, and hard limiting the MUD output of the BS closest to the MS

$$
\hat{\mathbf{v}}_{i}=\operatorname{sign}\left\{\boldsymbol{\Lambda}_{i}^{\left(m^{\prime}\right)}\right\} \text {. }
$$

d) Combining the SISO MUD outputs of the three BSs that serve the cell according to (26)

$$
\hat{\mathbf{v}}_{i}=\operatorname{sign}\left\{\sum_{m=1}^{M} \boldsymbol{\Lambda}_{i}^{(m)}\right\} .
$$

Two scenarios are considered. In the first scenario, the $K$ mobiles are all located in the center of the cell, and thus, the average power received at the three BSs are equal. Such a scenario is interesting from a theoretical perspective but does not reflect the behavior of actual cellular systems. In the second scenario, the $K$ MSs are located randomly within the cell, which is a more realistic assumption. The number of mobiles was varied from $K=1$ to 9 . In all simulations, enough trials are run to generate 100 independent error events.

\section{A. Mobiles in Center of Cell}

In the first test scenario, all $K$ cochannel mobiles are located in the center of the cell. This represents a worst-case situation for the conventional reception system (a), since the received carrier-to-interference power ratio (CIR) averaged over the $M$ BSs is minimized over all possible MS placements. In particular, the CIR at each BS is [17]

$$
\frac{C}{I}=\frac{1}{K-1}
$$

which is prohibitive even for $K=2$. However, the test scenario is a best-case situation for the proposed distributed MUD technique (d). This is for two reasons. First the signal transmitted by any one MS will be received with equal power at all three BSs, which will lead to the best macrodiversity improvement of all possible MS placements (see Appendix D of [18] for an illustration of this property); second, the $K$ signals received at each BS 


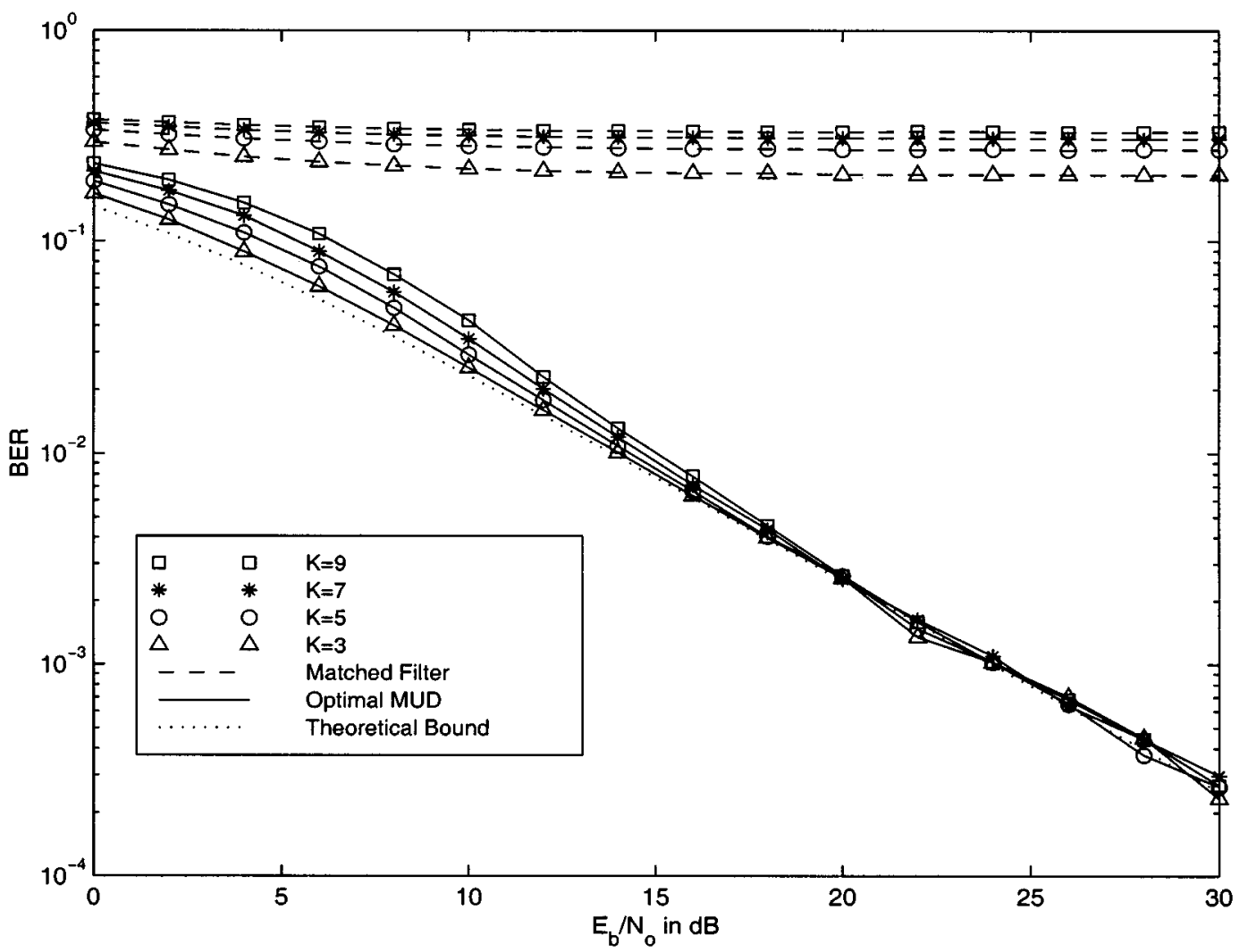

Fig. 2. Performance of conventional MF reception and MUD in fully-interleaved Rayleigh flat-fading with $K$ mobiles placed in the center of the cell and only one receiver used.

have equal power, and thus, the near-far problem is avoided. The reason that such a MS placement can be a worst case scenario for the conventional technique (a) and a best-case scenario for the distributed MUD technique (d) can be intuitively explained as follows. With conventional reception, signals originating from transmitters other than the desired transmitter are regarded as interference and, thus, small carrier to interference levels are to be avoided; however, with MUD, the transmissions from all users are regarded as useful information, and thus it is desirable for the received signals of all users to have identical power.

The performance of the conventional matched filter (a) and multiuser detector (c) is shown in Fig. 2. In each case, the hard bit decision is made using the output from the BS that is closest to the MS (i.e., macrodiversity is not used). ${ }^{2}$ For the conventional MF receiver, the bit-error performance is extremely poor. The bit-error ratio (BER) quickly flattens out, reaching a BER floor of between 0.2 and 0.3 . The floor rises with increasing $K$, but in all cases is unacceptable. The performance using MUD is much improved and for high SNR approaches the performance of the single user system (derived in Appendix D of [18]). For lower SNRs, performance degrades with increasing $K$. However, even for $K=9$, performance is within $5 \mathrm{~dB}$ of the single user bound at low SNR (i.e., in the region $P_{b}=10^{-1}$ ).

The performance of the two macrodiversity combining techniques is shown in Fig. 3. For the matched filter results (b), the outputs of the three BSs are MRC. For the MUD results (d),

${ }^{2}$ Since the mobiles are centrally located, any one of the three BSs can be used for the decision. the LLRs produced by the MUDs at the three BSs are added. For the MRC-MF system (b), performance is improved slightly compared to the MF receiver at only the closest BS (a). However, there is a BER floor on the order $10^{-1}$, and thus, performance is unacceptable for all $K>1$. The performance for the distributed MUD system (d) is dramatically superior to that of the system that uses the MUD output from the closest BS (c). Most of this improvement is due to the diversity effect of the system, as the performance bound for three-branch diversity is about $20 \mathrm{~dB}$ superior to the single receiver at $P_{b}=10^{-3}$ [19]. Performance for $K=3$ is close to the theoretical bound for three-branch diversity, but degrades with increasing $K$. Performance comes closer to the bound with increasing SNR, although not as quickly as with the single BS case. However, even for $K=9$, performance is within $2 \mathrm{~dB}$ of the single-user bound at $P_{b}=10^{-4}$.

A different representation of the simulated data is shown in Fig. 4. Here $E_{b} / N_{o}=10 \mathrm{~dB}$ and the number of transmitters is varied from $K=1$ to 9 . The performance of all four reception techniques is shown. When $K=1$ the performance of the conventional MF is identical to the performance of the MUD, with diversity reception offering a BER reduction of about two orders of magnitude. As $K$ increases, the performance of the conventional MF both with and without macrodiversity combining quickly deteriorates, and for large $K$ the BER with macrodiversity is about half that without. For MUD at the closest BS, the BER rises only slightly with increasing $K$. For distributed MUD, the BER increases at a slightly faster rate, but acceptable performance is still achieved with $K=9$. 


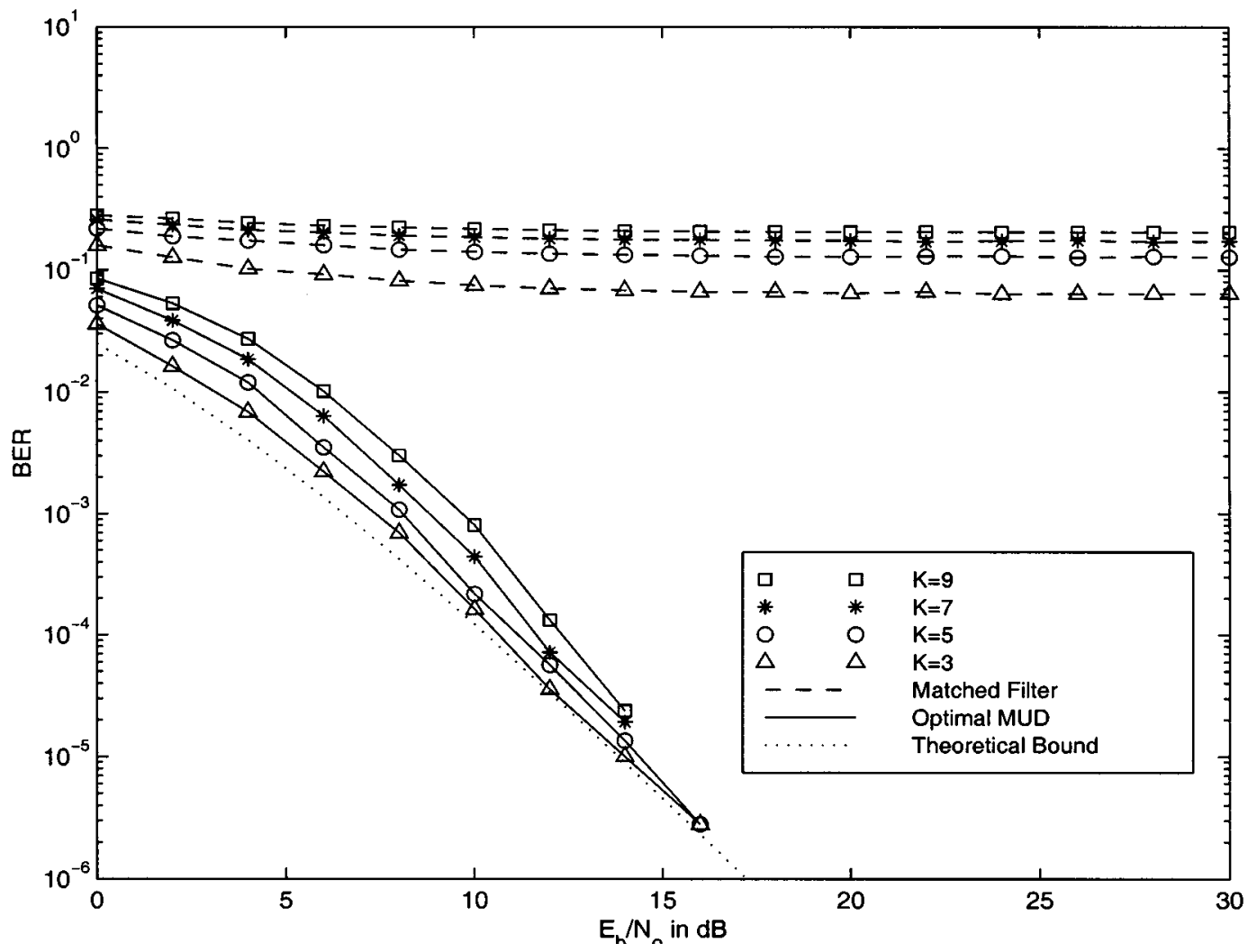

Fig. 3. Performance of conventional MRC macrodiversity and distributed MUD in fully-interleaved Rayleigh flat-fading with $K$ mobiles placed in the center of the cell and the outputs of all three receivers diversity combined.

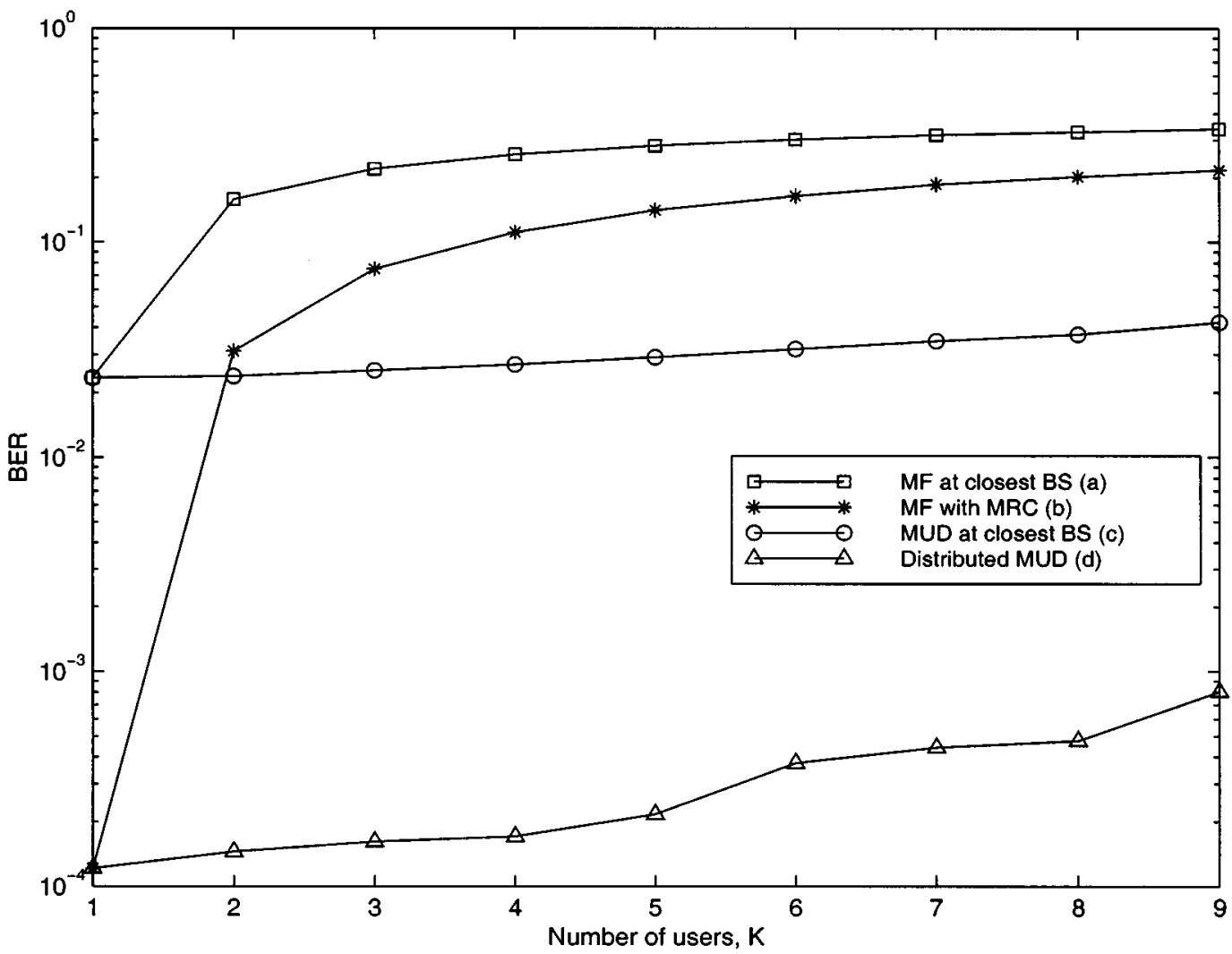

Fig. 4. Performance as a function of $K$ for four reception techniques with $K$ mobiles placed in the center of the cell and $E_{b} / N_{o}=10 \mathrm{~dB}$. 


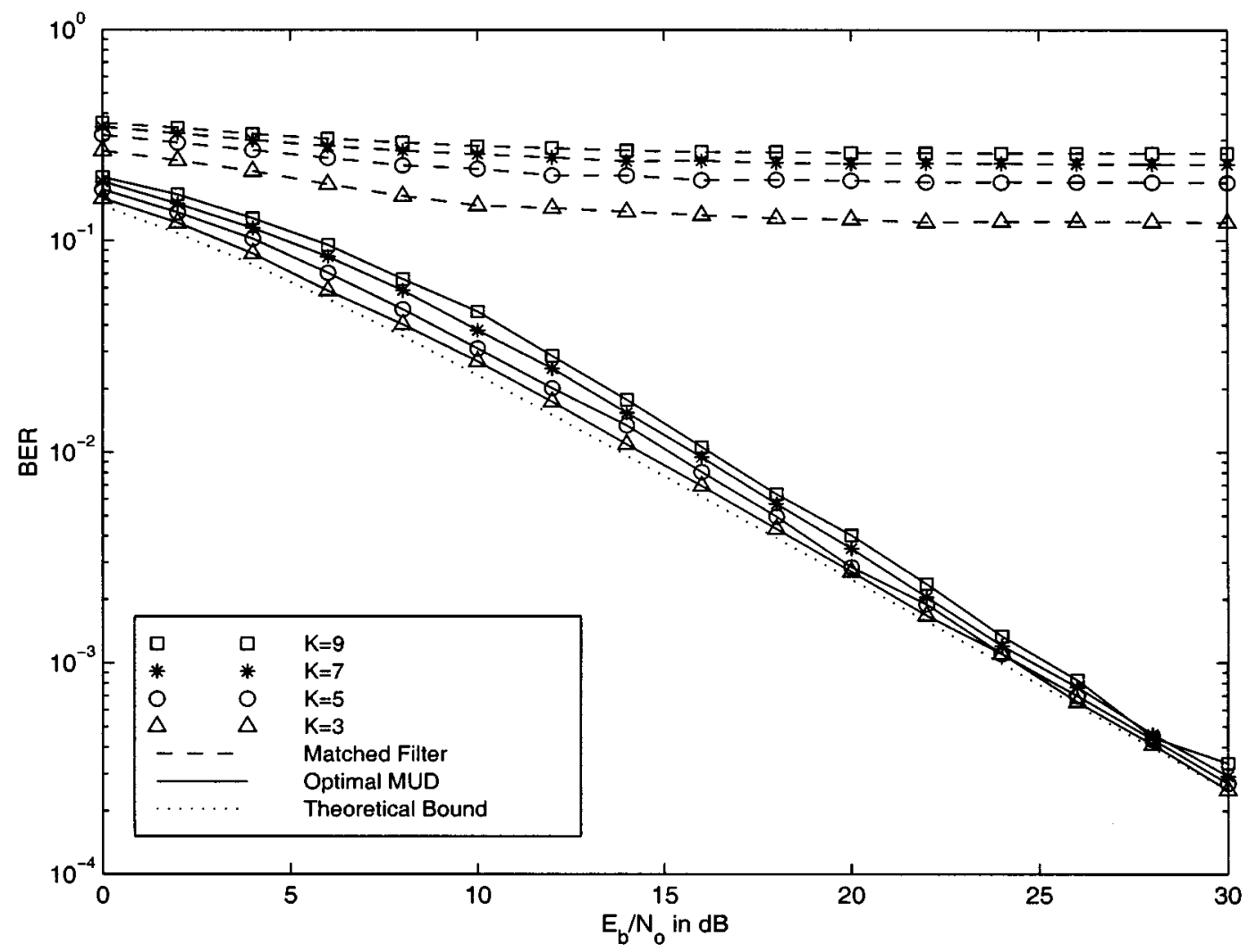

Fig. 5. Performance of conventional MF reception and MUD in fully-interleaved Rayleigh flat-fading with $K$ randomly placed mobiles and only one receiver used.

\section{B. Mobiles Randomly Placed in Cell}

In the second test scenario, the $K$ cochannel mobiles are randomly placed within the hexagonal cell. After each Monte Carlo trial consisting of one TDMA frame from each transmitter, the mobiles are randomly repositioned. The mobiles are power controlled by the BS closest to it such that the average SNR at the closest BS is $E_{s} / N_{o}$. Define the average SNR of MS $k$ received at $\mathrm{BS} m$ as

$$
\bar{\gamma}_{m, k}=\frac{E_{s}}{N_{o}} E\left\{\left|c_{m, k}(t)\right|^{2}\right\} .
$$

Then, the power control will ensure that $E\left\{\left|c_{m^{\prime}, k}(t)\right|^{2}\right\}=1$, where $m^{\prime}$ is the BS closest to MS $k$. The SNR at the other two BSs will depend on the geometry of the MS and BS placements and the path loss exponent. If the three BSs are placed on a normalized plane at coordinates $(-\sqrt{1 / 3}, 1),(\sqrt{4 / 3}, 0)$, and $(-\sqrt{1 / 3},-1)$, and MS $k$ is placed at coordinate $(x, y)$, then the distance $d_{m, k}$ between BS $m$ and MS $k$ will be

$$
\begin{aligned}
& d_{1, k}=\left[\left(\sqrt{\frac{1}{3}}+x\right)^{2}+(1-y)^{2}\right]^{1 / 2} \\
& \left.\left.d_{2, k}=\left[\left(\sqrt{\frac{4}{3}}-x\right)^{2}+y^{2}\right]^{1 / 2}\right]^{2}\right]^{2} .
\end{aligned}
$$

The received SNRs will then be

$$
\begin{aligned}
\bar{\gamma}_{1, k} & =\frac{E_{s}}{N_{o}}\left(\frac{d_{m^{\prime}, k}}{d_{1, k}}\right)^{n_{e}} \\
\bar{\gamma}_{2, k} & =\frac{E_{s}}{N_{o}}\left(\frac{d_{m^{\prime}, k}}{d_{2, k}}\right)^{n_{e}} \\
\bar{\gamma}_{3, k} & =\frac{E_{s}}{N_{o}}\left(\frac{d_{m^{\prime}, k}}{d_{3, k}}\right)^{n_{e}}
\end{aligned}
$$

where $m^{\prime}$ is the BS closest to MS $k$ and $n_{e}$ is the path loss exponent. For free space, $n_{e}=2$. However, field measurements have shown that the exponent varies considerably in outdoor cellular systems, and for this experiment we chose $n_{e}=3$ [20].

This scenario is better than the first for the conventional reception system (a), since the CIR will, on average, be higher. However, the performance of the distributed MUD system (d) will not be as impressive as it was in the first scenario. This is because now the signal from each BS is no longer received with equal power at the three BSs, and thus, the improvement from macrodiversity combining is reduced. Also, the signal powers of the $K$ signals received by each BS are no longer equal, and thus, the near-far problem is not necessarily avoided.

The performance of the conventional matched filter (a) and multiuser detector (c) is shown for this scenario in Fig. 5. These results were generated by making a hard bit decision using the output from the BS that is closest to the MS (i.e., macrodiversity is not used). For the conventional MF receiver, the biterror performance is once again very poor. The BER reaches a floor, which is only slightly lower than the floor reached in 


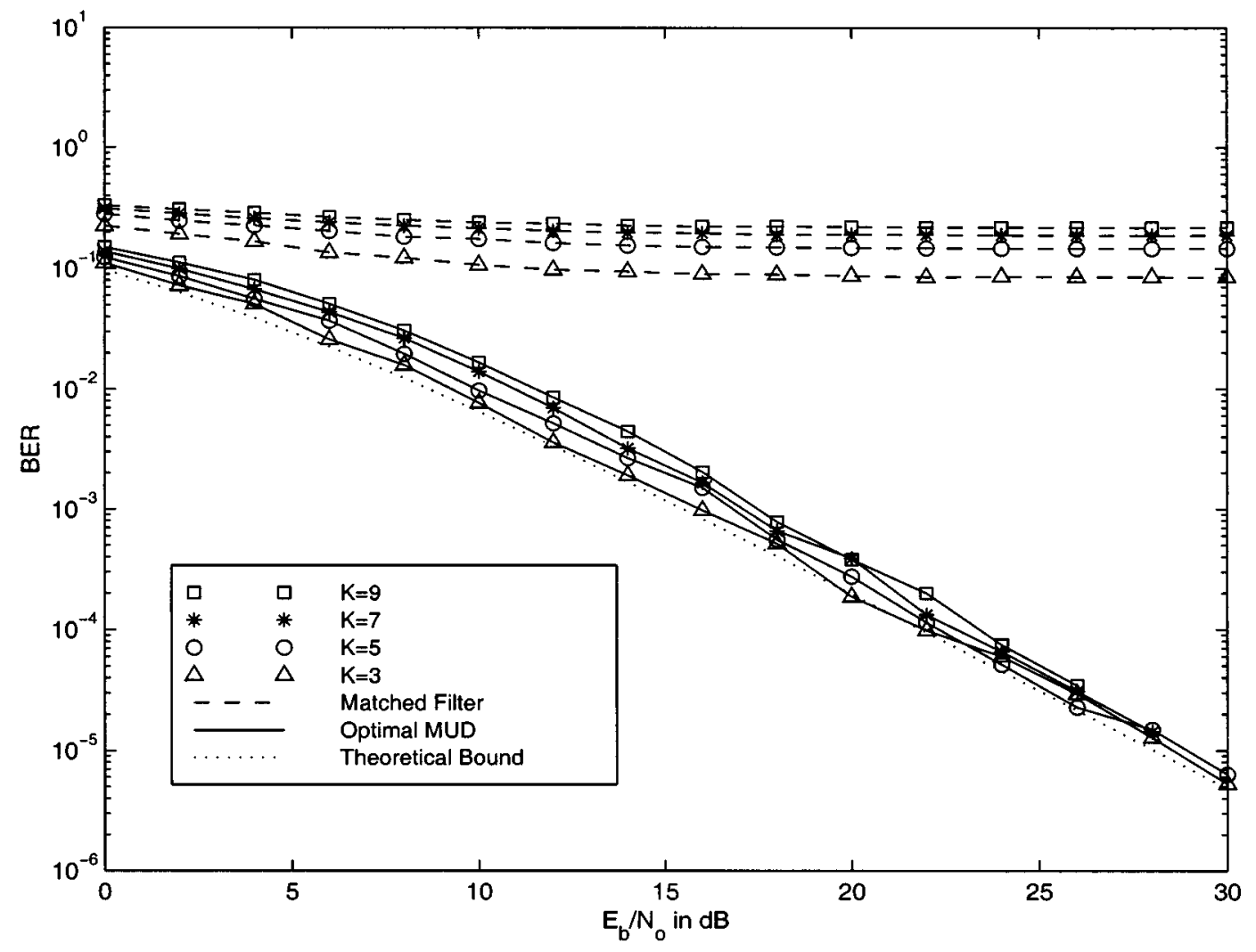

Fig. 6. Performance of conventional MRC macrodiversity and distributed MUD in fully-interleaved Rayleigh flat-fading with $K$ randomly mobiles placed and the outputs of all three receivers diversity combined.

the equal power case of scenario one. The reduced BER floor can be attributed to increased average CIR. The performance is improved by using MUD, with performance again degrading with increasing $K$. For high SNR, the performance approaches that of the single-user case, although this convergence occurs at higher SNR than was observed in scenario one. This can be attributed to the imbalance of received powers at each BS.

The performance of the two macrodiversity combining techniques for this scenario is shown in Fig. 6. For the matched filter results (b), the outputs of the three BSs are maximal ratio combined. For the MUD results (d), the LLRs produced by the MUDs at the three BSs are added. Again, we observe improved performance for the MRC-MF system, although the absolute performance is comparable to the same system under scenario one. The performance for the distributed MUD system is superior to that of the system that uses the MUD output from only the closest BS. However, the improvement is much less dramatic than that observed for the same reception technique under scenario one. This is primarily due to the fact that the macrodiversity effect is less beneficial when the signal from each MS is received with different powers at the three BSs. Nevertheless, an improvement of about $8 \mathrm{~dB}$ can be achieved at $P_{b}=10^{-3}$ by using macrodiversity (compared to MUD without macrodiversity). Again, performance degrades with increasing $K$, but converges to the single-user bound for high SNR.

In Fig. 7, the number of transmitters is varied from $K=1$ to $K=9$, while $E_{b} / N_{o}$ is held at the constant value of $20 \mathrm{~dB}$. The performance of all four reception techniques is shown. For
$K=1$, macrodiversity reception provides an order of magnitude reduction of BER. Again, we see that as $K$ increases, the performance of the conventional MF both with and without macrodiversity combining quickly deteriorates. For MUD at the closest BS, the BER rises slightly with increasing $K$. For distributed MUD, the BER increases at a slightly faster rate, but acceptable performance is still achieved with $K=9$.

\section{COOPERATIVE DECODING FOR TDMA NETWORKS}

When the transmission is encoded by an FEC code, it is possible pass the output of a SISO FEC decoder back to the MUD algorithm in an iterative manner. A proposed cooperative decoding architecture is shown in Fig. 8. At receiver $m$, the received signal $y_{m}(t)$ is passed through a bank of $K$ matched filters, each matched to and synchronized with one of the transmitters. The matched filter output $\mathbf{y}^{(m)}$ is passed to a SISO multiuser detector, which produces the LLR $\Lambda_{m, \text { mud }}^{(q)}$ (the superscript $q$ now denotes the iteration number). The a priori information $\mathbf{z}_{\text {mud }}^{(q)}$ is subtracted from the LLR output of the MUD to produce the extrinsic information

$$
\overline{\mathbf{\Psi}}_{m}^{(q)}=\Lambda_{m, \text { mud }}^{(q)}-\mathbf{z}_{\text {mud }}^{(q)}
$$

The extrinsic information from the $M$ multiuser detectors are then combined according to

$$
\overline{\boldsymbol{\Psi}}^{(q)}=\sum_{m=1}^{M} \overline{\mathbf{\Psi}}_{m}^{(q)}
$$




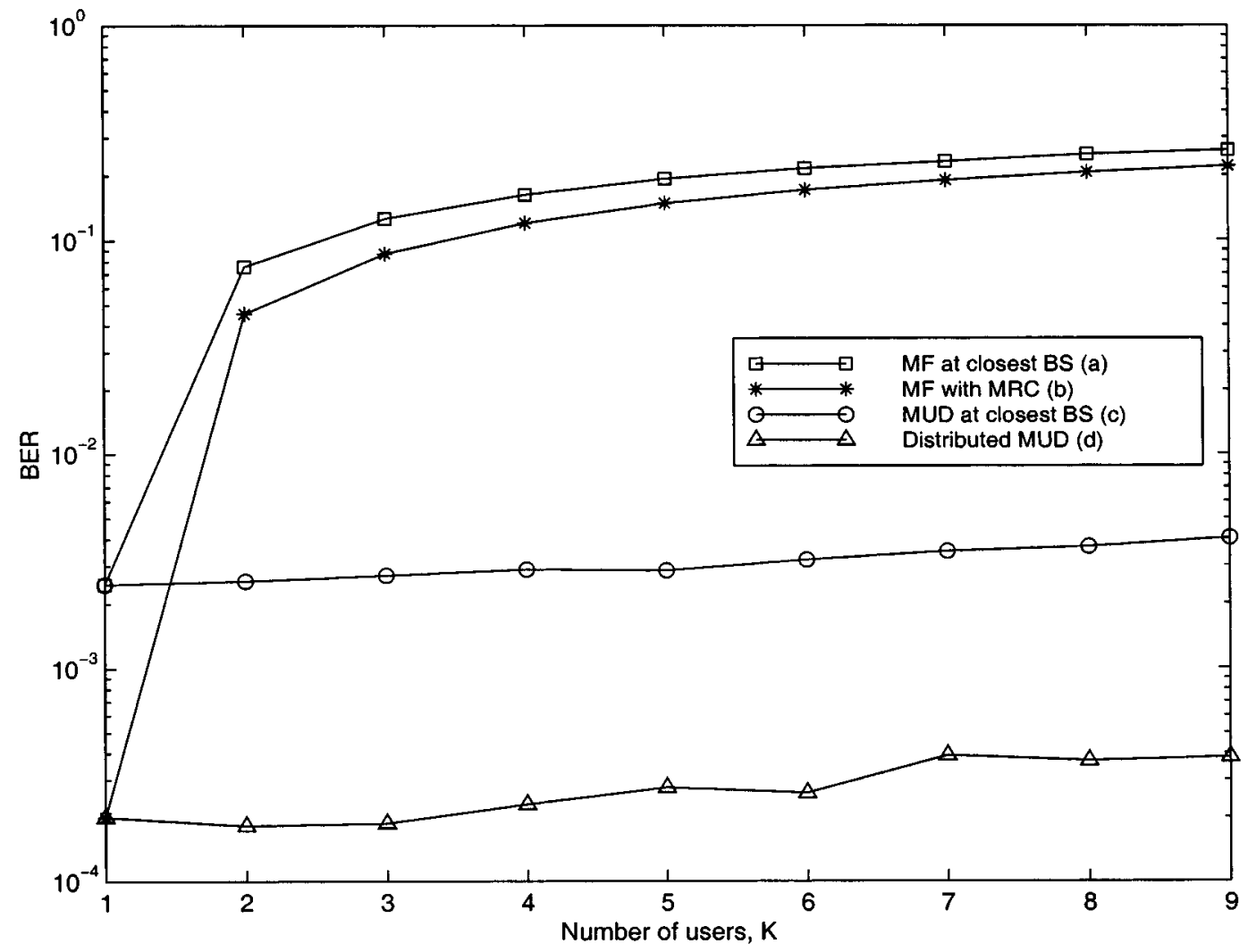

Fig. 7. Performance as a function of $K$ for four reception techniques with $K$ randomly placed mobiles and $E_{b} / N_{o}=20 \mathrm{~dB}$.

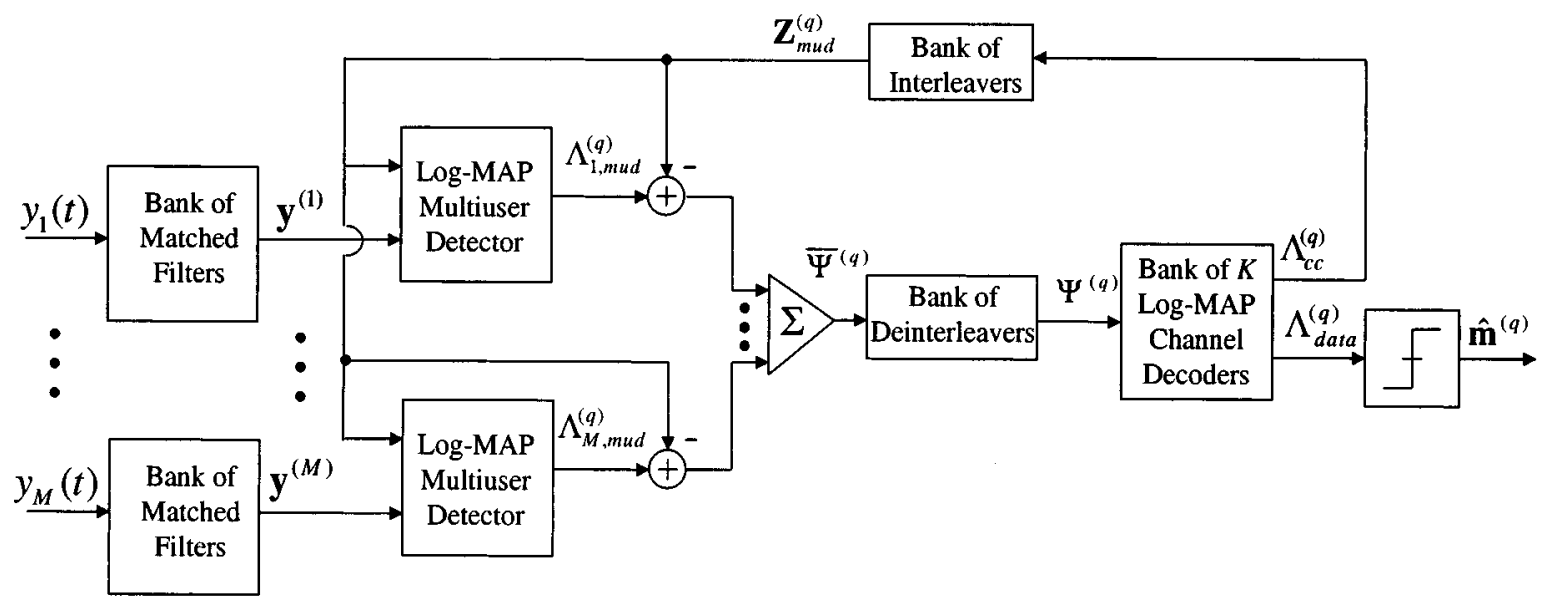

Fig. 8. Architecture for TDMA cooperative decoding.

Next, $\overline{\mathbf{\Psi}}^{(q)}$ is deinterleaved and decoded by a bank of $K$ SISO channel decoders, each implemented with the log-MAP algorithm [16]. The channel decoders produce $\boldsymbol{\Lambda}_{c c}^{(q)}$, the LLR of the code bits after decoder iteration $q$. This LLR is reinterleaved to form the a priori input to the multiuser detectors, $\mathbf{z}_{\text {mud }}^{(q)}$. Finally after $Q$ iterations, an estimate of the data bits $\hat{\mathbf{m}}^{(Q)}$ is produced by limiting the LLR of the data bits $\Lambda_{\text {data }}^{(Q)}$.

\section{Simulation Study: Coded CASE}

Consider a coded TDMA network composed of $K$ transmitters and $M=3$ receivers, which models a TDMA cellular system with 120-degree sectorized antennas. Each transmitter uses a rate $1 / 2$, constraint length 3 convolutional code with octal generators $(7,5)$. The size of the data frame is $L=264$, of which the last two bits are zeros used to terminate the trellis of the encoder. All transmitters use the same 24 by 22 block interleaver, and the channel is (fully-interleaved) Rayleigh flat-faded. We again assume that the $K$ asynchronous BPSK signals arrive at each receiver with equally spaced delays according to (27). Enough Monte Carlo trials were run to generate 50 frame errors.

\section{A. Mobiles in Center of Cell}

For the first scenario, all $K$ transmitters are located at the center of the cell. Thus, the average power of all users is the 


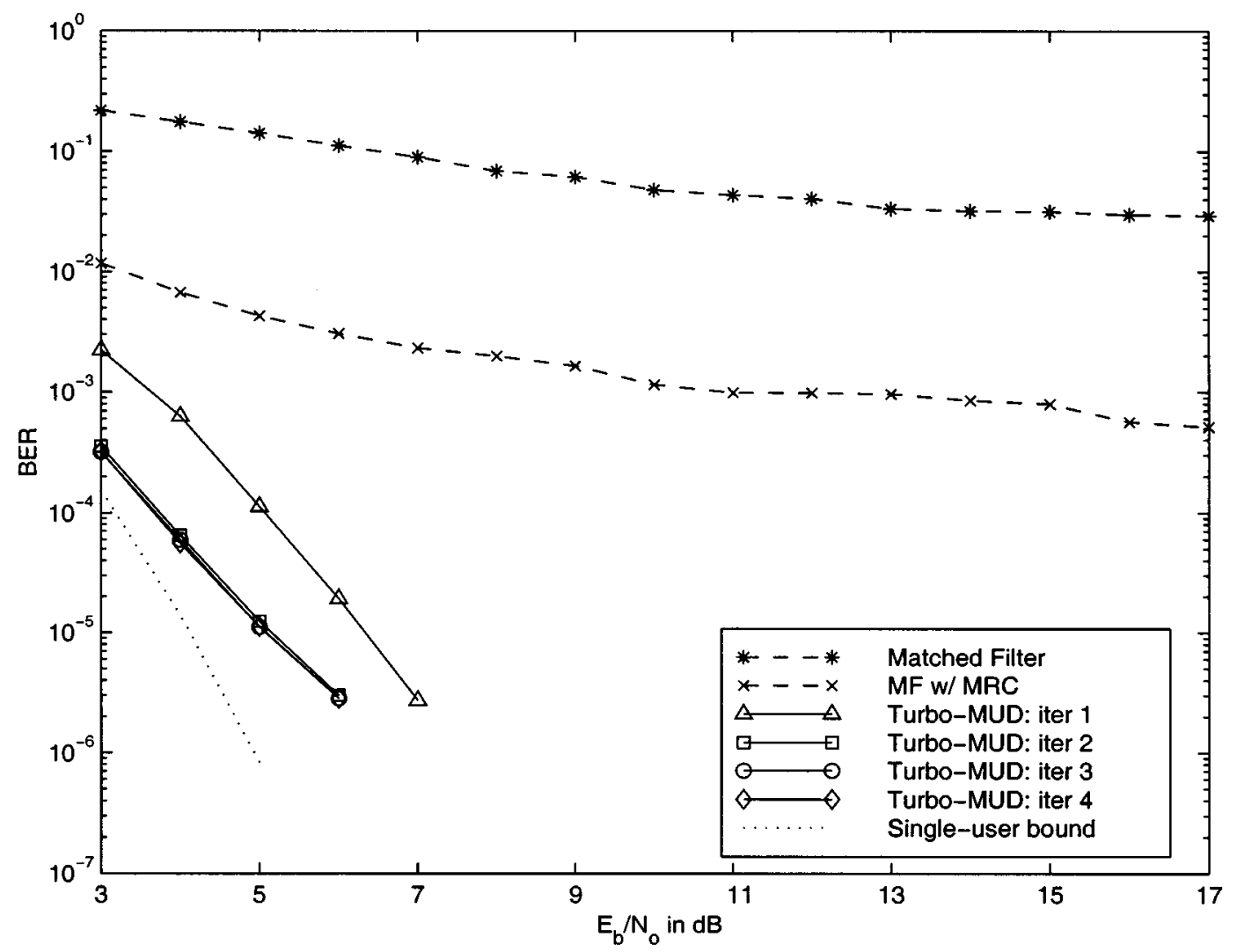

Fig. 9. Bit-error performance of a TDMA system with $K=3$ centrally located transmitters and $M=3$ receivers in a Rayleigh flat-fading channel with rate $r=1 / 2$, constraint length 3 convolutional coding as parameterized by decoding technique.

same at all $M$ receivers, i.e., $E\left\{\left|c_{m, k}(t)\right|^{2}\right\}=1, \forall m, k$. Simulation results are shown for this scenario with $K=3$ users in Fig. 9. The upper curve shows the performance of the conventional system, which utilizes a matched filter at each BS and makes the bit decisions using the MF output of the BS closest to the mobile (i.e., macrodiversity is not used). For the next curve down, the MF outputs of the three receivers are combined according to (24); for this case macrodiversity combining is used, but multiuser detection is not used. By using macrodiversity, a performance improvement of about 1.5 orders of magnitude in BER reduction is observed. The lowest curve (dotted line) shows the performance of a MRC combined single user system (i.e., $K=1$ ), while the four solid lines show the performance of the iterative architecture. There is a $1-\mathrm{dB}$ performance gain from the first to the second iteration of decoding but no noticeable improvement after the second iteration. After the second iteration, the performance is within about $1 \mathrm{~dB}$ of the single user system at $P_{b}=10^{-5}$.

In Fig. 10, the BER is shown as a function of the number of users, $1 \leq K \leq 9$. There are again $M=3$ receivers and the channel is Rayleigh flat-faded. In this case, the SNR is held to the constant value of $E_{b} / N_{o}=5 \mathrm{~dB}$. For the two methods that directly use the matched filter outputs, performance degrades quickly with increasing $K$. Performance also degrades with increasing $K$ for the iterative decoding method, although performance is always better than when the MF outputs are used without multiuser detection. For $K>1$, almost all of the performance gain is achieved after the second iteration. There is only a slight gain from the second to the third iteration and again from the third to the fourth.

\section{B. Mobiles Randomly Placed in Cell}

In actual TDMA systems, the mobiles are not all centrally located. For the second scenario, the $K$ transmitters were placed randomly within the hexagonal cell. After each Monte Carlo simulation (one $N$ symbol packet from each of the $K$ transmitters), the mobiles were placed in new locations at random. The mobiles were power controlled such that $E\left\{\left|c_{m^{\prime}, k}(t)\right|^{2}\right\}=1$, where $m^{\prime}$ is the BS that is closest to MS $k$. The power at the other two BSs was found using (37)-(39) and a loss exponent of $n_{e}=3$.

Simulation results are shown for $K=3$ uniformly distributed users in Fig. 11. For the upper curve, the bit decisions are made using the MF output of the BS closest to the corresponding MS. The relative gain by macrodiversity combining the MF outputs is less than was observed for the scenario with centrally located MSs. Also, the performance of the single-user system is worse than was observed in the previous scenario. This is because the total signal power received by the three BSs is less when the mobiles are uniformly distributed than when they are centrally located. For this scenario, the performance of the iterative receiver comes very close to the single-user bound. As before, most of the performance gain comes from the first to the second iterations. However, now a noticeable improvement can be observed from the second to the third iteration and again for the third to the fourth iteration. After four iterations, performance is within 


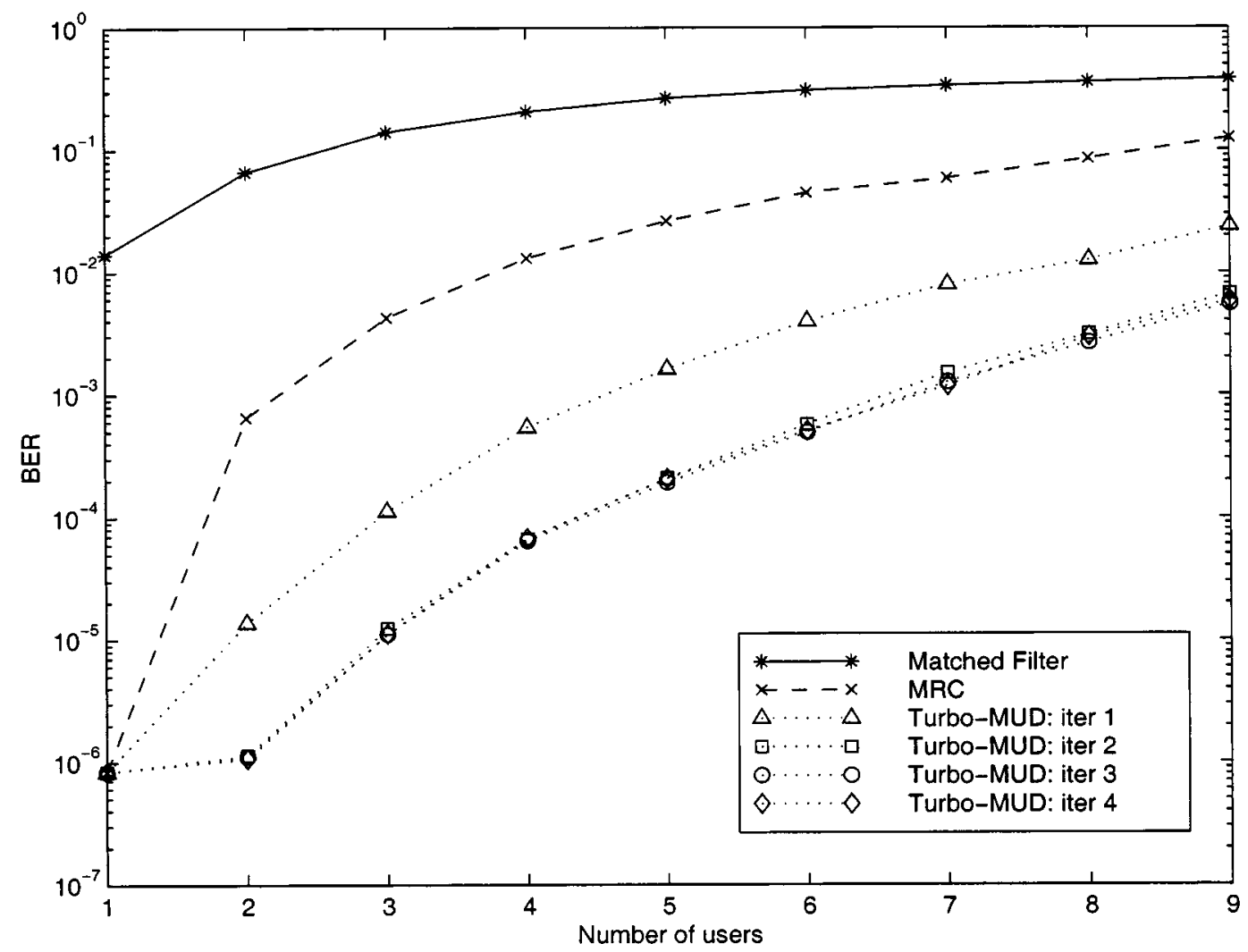

Fig. 10. Bit-error performance of a TDMA system in a Rayleigh flat-fading channel with rate $r=1 / 2$, constraint length 3 convolutional coding for $1 \leq K \leq 9$ centrally located transmitters, $M=3$ receivers, and $E_{b} / N_{o}=5 \mathrm{~dB}$.

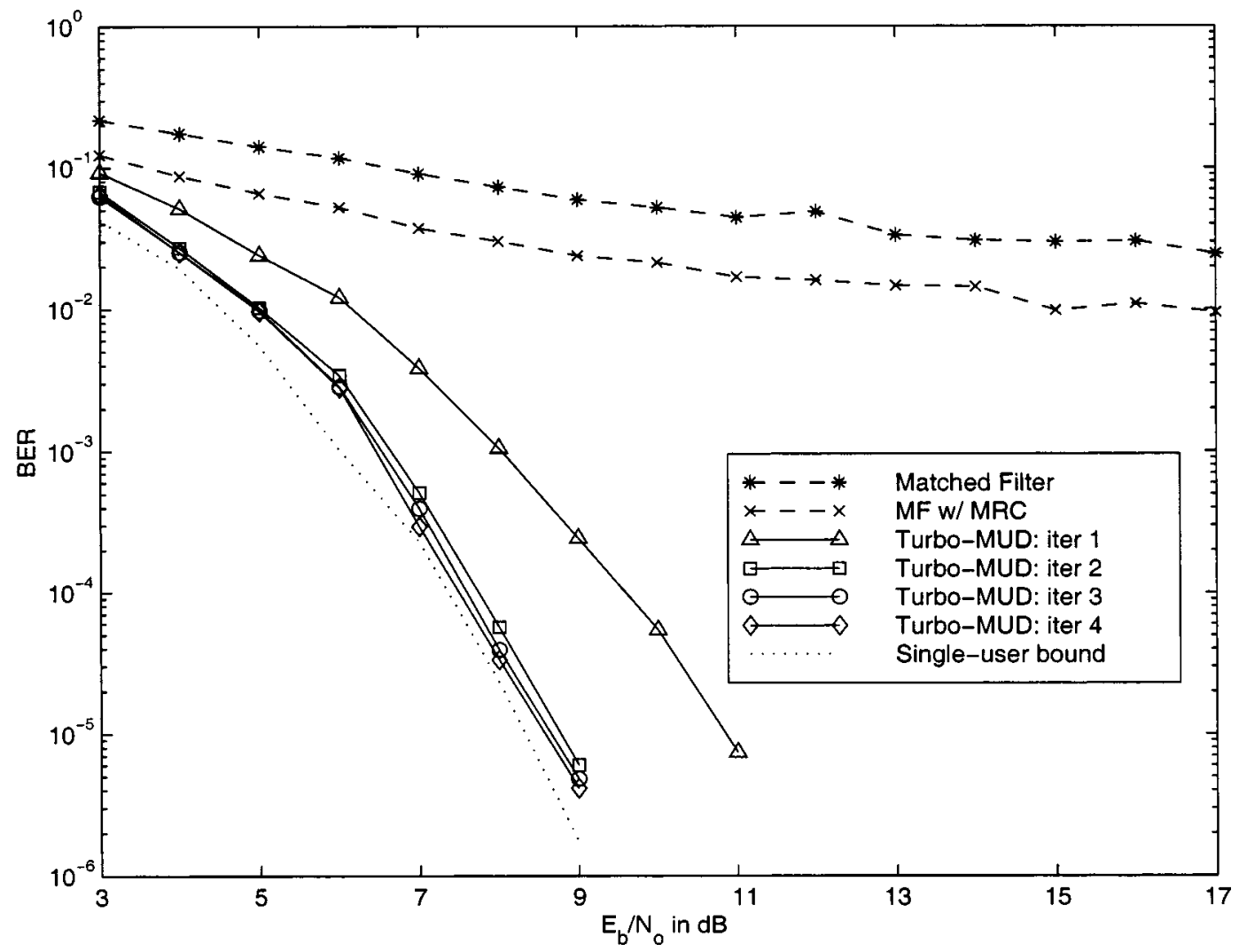

Fig. 11. Bit-error performance of a TDMA system with $K=3$ uniformly distributed transmitters and $M=3$ receivers in a Rayleigh flat-fading channel with rate $r=1 / 2$, constraint length 3 convolutional coding as parameterized by decoding technique. 


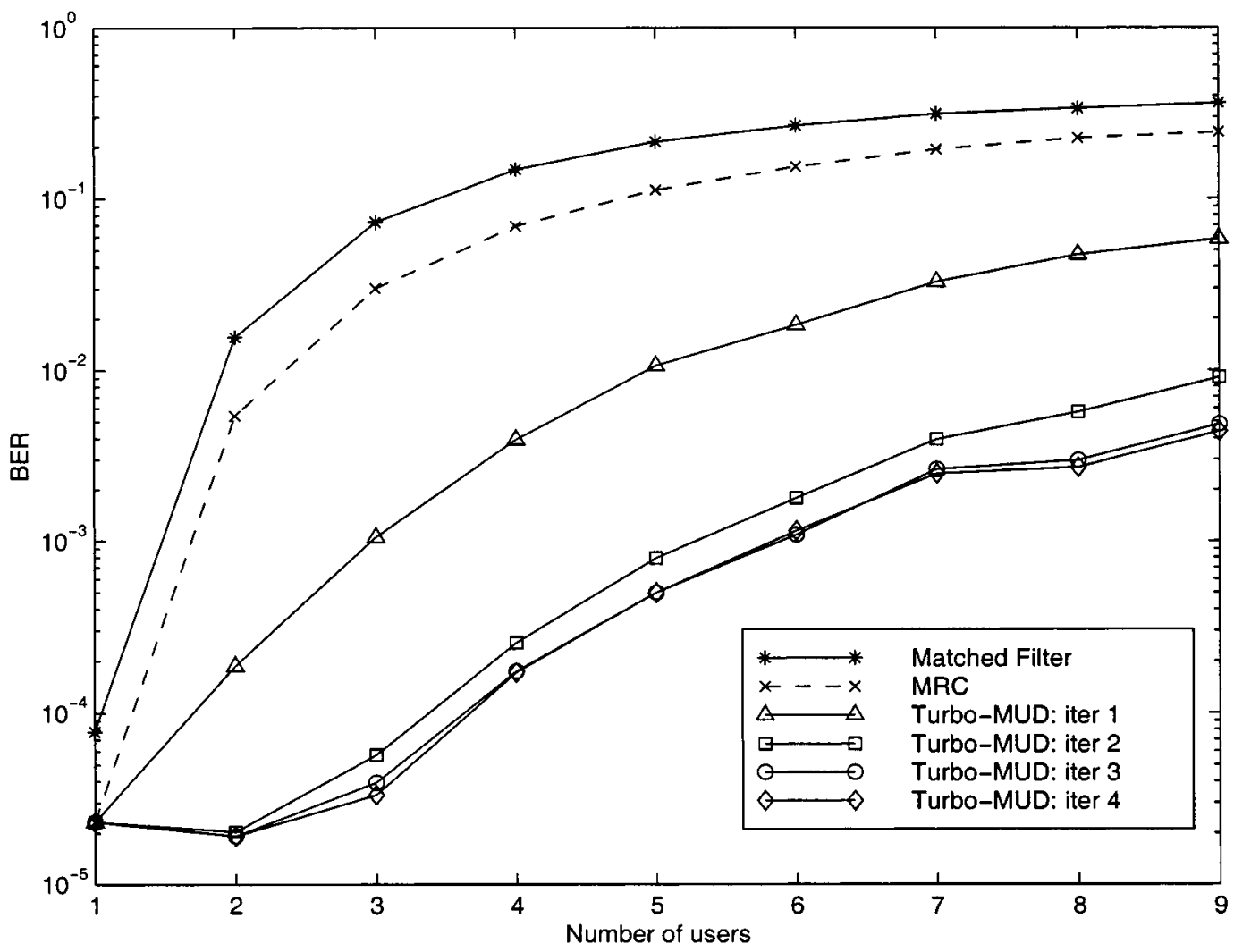

Fig. 12. Bit-error performance of a TDMA system in a Rayleigh flat-fading channel with rate $r=1 / 2$, constraint length 3 convolutional coding for $1 \leq K \leq 9$ uniformly distributed transmitters, $M=3$ receivers, and $E_{b} / N_{o}=8 \mathrm{~dB}$.

$0.2 \mathrm{~dB}$ of the single-user bound. This is encouraging, particularly since this scenario is more realistic than the previous scenario which had shown a larger gap between the performance of the iterative technique and that of the single-user system.

In Fig. 12, the BER is shown as a function of the number of users, $1 \leq K \leq 9$. There are again $M=3$ receivers and the channel is Rayleigh flat-faded. The SNR is held to the constant value of $E_{b} / N_{o}=8 \mathrm{~dB}$. For the two methods that directly use the matched filter outputs, performance degrades quickly with increasing $K$. Performance also degrades with increasing $K$ for the iterative decoding method, although performance is always better than when multiuser detection is not used. While most of the performance gain is achieved from the first to the second iteration, there is a noticeable gain from the second to the third iteration. There is little, if any, gain from the third to the fourth iteration.

\section{CONCLUSION}

The combination of soft-output multiuser detection, macrodiversity combining, and iterative decoding is an effective method for increasing the capacity of TDMA cellular networks. Because the number of cochannel interferers is typically rather small, it is reasonable to use the optimal soft-output multiuser detection algorithm presented in this paper, even though its complexity is exponential in the number of users. When iterative decoding is employed, a significant performance gain is improved after just two iterations. Because of the macrodiversity effect, performance is particularly good at the center of edge excited cells, which is normally where performance is the worst when conventional techniques are used.

The simulations assumed that the complex channel gains for each user are known at each receiver, and that perfect timing and carrier synchronization is achieved for each user at each receiver. While it is possible to obtain reliable channel information for the closest (most powerful) user at each BS, obtaining reliable channel information for the more distant (less powerful) users is more problematic. Thus, the topic of channel estimation and synchronization must be further addressed before this technique can be practically applied, and is a topic of ongoing research. Furthermore, because the proposed technique requires soft-values to be passed over the backhaul link to a BSC, the impact of the number of bits of quantization of the various soft-values should be studied.

\section{REFERENCES}

[1] M. Moher, "An iterative multiuser decoder for near-capacity communications," IEEE Trans. Commun., vol. 46, pp. 870-880, July 1998.

[2] P. D. Alexander, M. C. Reed, J. A. Asenstorfer, and C. B. Schlegel, "Iterative multiuser interference reduction: Turbo CDMA," IEEE Trans. Commun., vol. 47, pp. 1008-1014, July 1999.

[3] C. Berrou, A. Glavieux, and P. Thitimasjshima, "Near shannon limit error-correcting coding and decoding: Turbo-codes(1)," in Proc. IEEE Int. Conf. Communications, Geneva, Switzerland, May 1993, pp. $1064-1070$

[4] S. J. Grant and J. K. Cavers, "Performance enhancement through joint detection of cochannel signals using diversity arrays," IEEE Trans. Commun., vol. 46, pp. 1038-1049, Aug. 1998.

[5] M. C. Valenti and B. D. Woerner, "Combined multiuser reception and channel decoding for TDMA cellular systems," in Proc. IEEE Vehicular Technology Conf., Ottawa, ON, Canada, May 1998, pp. 1915-1919. 
[6] J.-T. Chen, J. Liang, H.-S. Tsai, and Y.-K. Chen, "Joint MLSE receiver with dynamic channel description," IEEE J. Select. Areas Commun., vol. 16, pp. 1604-1615, Dec. 1998.

7] Z. J. Haas and C.-P Li, "The multiply-detected macrodiversity scheme for wireless cellular systems," IEEE Trans. Veh. Tech., vol. 47, pp. 506-530, May 1998.

[8] B. R. Vojcic, Y. Shama, and R. L. Pickholtz, "Optimum soft output MAP detector for coded multiuser communications," in Proc. IEEE Int. Symp. Information Theory, Ulm, Germany, June 1997, p. 229

[9] S. Verdú, "Minimum probability of error for asynchronous gaussian multiple-access channels," IEEE Trans. Inform. Theory, vol. 32, pp. 85-96, Jan. 1986.

[10] L. R. Bahl, J. Cocke, F. Jelinek, and J. Raviv, "Optimal decoding of linear codes for minimizing symbol error rate," IEEE Trans. Inform. Theory, vol. 20, pp. 284-287, Mar. 1974

[11] M. C. Valenti, "An optimal soft-output multiuser detection algorithm and its applications," in Proc. Int. Symp. Turbo Codes and Related Topics, Brest, France, Sept. 2000, pp. 177-180.

[12] M. C. Valenti and B. D. Woerner, "Multiuser detection with base station diversity," in Proc. IEEE Int. Conf. Universal Personal Communications, Florence, Italy, Oct. 1998, pp. 1189-1194.

[13] — , "Distributed multiuser detection for the TDMA cellular uplink," Electron. Lett., vol. 35, pp. 1803-1804, 1999.

[14] — - "Combined multiuser detection and channel decoding with receiver diversity," in Proc. IEEE GLOBECOM, Communication Theory Mini-Conf., Sydney, Australia, Nov. 1998, pp. 137-142.

[15] S. Verdú, Multiuser Detection. Cambridge, U.K.: Cambridge Univ. Press, 1998.

[16] P. Robertson, P. Hoeher, and E. Villebrun, "Optimal and sub-optima maximum a posteriori algorithms suitable for turbo decoding," Eur Trans. Telecommun., vol. 8, pp. 119-125, Mar. 1997.

[17] K. S. Gilhousen, I. M. Jacobs, R. Padovani, J. L. A. Weaver, and C. E. Whitley, "On the capacity of a cellular CDMA system," IEEE Trans. Veh. Technol., vol. 40, pp. 303-312, May 1991

[18] M. C. Valenti, "Iterative detection and decoding for wireless communications," Ph.D., Bradley Dept. Elec. and Comp. Eng. Virginia Institute of Technology, Blacksburg, Virginia, 1999.

[19] G. L. Stüber, Principles of Mobile Communication. Norwell, MA Kluwer, 1996.

[20] S. Y. Seidel, T. S. Rappaport, S. Jain, M. Lord, and R. Singh, "Path loss, scattering and multipath delay statistics in four european cities for digital cellular and microcellular radiotelephone," IEEE Trans. Veh. Technol., vol. 40, pp. 721-730, Nov. 1991.

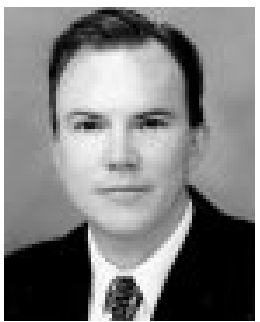

Matthew C. Valenti (M'99) received the B.S.E.E. degree from Virginia Institute of Technology (Virginia Tech), Blacksburg, in 1992, the M.S.E.E. degree from The Johns Hopkins University, Baltimore, MD, in 1995, and the Ph.D. degree in electrical engineering from Virginia Tech, in 1999, where he was a Bradley Fellow.

He is currently an Assistant Professor with the Department of Computer Science and Electrical Engineering, West Virginia University, Morgantown. His research interests are in the areas of communication theory, error correction coding, applied information theory, and wireless multiple-access networks. He also acts as a consultant to several companies engaged in various aspects of turbo codec design, including software radio, FPGA, and ASIC implementations for military, satellite, and third generation cellular applications. Prior to attending graduate school at Virginia Tech, he was an Electronics Engineer with the United States Naval Research Laboratory, Washington, DC, where he was engaged in the design and development of a spacebourne adaptive antenna array and a system for the collection and correlation of maritime ELINT signals.

Brian D. Woerner (M'91) was born on October 11, 1964. He received the B.S degree in computer and electrical engineering from Purdue University, West Lafayette, IN, in 1986, and the M.S. and Ph.D. degrees from the University of Michigan, Ann Arbor, in 1987 and 1991, respectively, where he was a Unisys Fellow. He also received the Master of Public Policy degree from the University of Michigan's School of Public Policy Studies, with an emphasis in telecommunications policy.

He is currently a Professor with the Bradley Department of Electrical Engineering, Virginia Tech, Blacksburg. He has served two two-year terms as Director of the Mobile and Portable Radio Research Group and is currently an Associate Director 